\section{Monitoring of foodborne pathogens in raw cow milk in Tuscany}

\author{
Laura Gasperetti, ${ }^{1}$ Alessia D'Alonzo, ${ }^{1}$ \\ Matteo Senese, ${ }^{1}$ Ilaria Fabbri, ${ }^{1}$ \\ Cristina Cirri, ${ }^{1}$ Carla Milioni, ${ }^{2}$ \\ Valeria Valenza, ${ }^{3}$ Rita Tolli, \\ Francesca Campeis, ${ }^{1}$ Roberto Fischetti ${ }^{1}$
}

'Istituto Zooprofilattico Sperimentale

delle Regioni Lazio e Toscana,

Laboratorio Alimenti Sezione di Pisa;

2Dipartimento di Scienze Agrarie

Alimentari e Agro Ambientali, Università

di Pisa; ${ }^{3}$ sstituto Zooprofilattico

Sperimentale delle Regioni Lazio e

Toscana, Centro di Riferimento Regionale

degli Enterobatteri Patogeni, Roma, Italy

\section{Abstract}

Raw milk consumption in Italy has increased over the last few years and although raw milk is characterised by cold chain, short shelf-life and the duty of boiling before domestic consumption, it is still considered a hazard. From 2010 to 2013 a monitoring survey of raw milk sold through vending machines was carried out to investigate the occurrence of several foodborne pathogens stipulated in the national legal requirements, i.e. Listeria monocytogenes, Campylobacter spp., Salmonella spp., Escherichia coli 0:157 and coagulase-positive Staphylococci. A total of 127 raw milk samples were collected from 19 dairy herds in Tuscany Region, Italy. In addition, the milk samples were tested for the presence and count of Yersinia genus. Results shown that only one sample was positive for non verocytotoxin-producing $E$. coli $0: 157$, whereas a total of 38 samples (29.9\%) were postive for Yersinia genus; of the total 39 isolated bacteria, $23.6 \%$ were $Y$. enterocolitica, $2.4 \% Y$. kristenseni and $4.7 \% Y$. frederiksenii. None isolate was enteropathogenic; serotypes 0:5 and 0:8 were found in 16.6 and $13.3 \%$ of the isolates respectively, whereas none of the serotypes tested was detected in $70 \%$ of the isolates. The most probable number method revealed a count value between 0.03 and $24 \mathrm{MPN} / \mathrm{mL}$. Based on these data a general assurance on health safety of raw milk produced and sold in Tuscany could be assessed.

\section{Introduzione}

L'interesse nel valutare la sicurezza del latte crudo, nasce dal fatto che, nell'ultimo decen- nio, il consumo di questo prodotto si è diffuso in maniera consistente nell'intero territorio nazionale. Il costo contenuto, il ridotto impatto ambientale ed il desiderio di naturalità e ritorno alle tradizioni hanno contribuito a consolidare una realtà produttiva, in precedenza pressoché assente in Italia. Dal 2008 (0M 10/12/2008) è obbligatoria la dicitura prodotto da consumarsi dopo bollitura; nonostante ciò il consumatore non sempre segue tale norma di comportamento e di conseguenza consuma il latte tal quale.

Nel territorio toscano ed in particolare in quello dell'area compresa tra le Province di Lucca, Livorno, Pisa e Massa-Carrara sono presenti numerose aziende produttrici di latte crudo destinato ai distributori automatici delle stesse zone; la cessione di latte crudo mediante vendita diretta o mediante macchine erogatrici è disciplinata in dettaglio dall'Intesa Stato Regioni del 25 gennaio 2007 (IS0, 2001). Al fine di delineare un quadro generale sulla sicurezza di tale prodotto, il presente progetto di ricerca ha voluto indagare in campioni di latte crudo prelevati alle macchine erogatrici 0 direttamente presso le aziende che effettuano vendita diretta (19 aziende controllate) la presenza sia dei patogeni previsti dalla normativa vigente (Listeria monocytogenes, Campylobacter spp., Salmonella spp., Escherichia coli 0:157 e stafilococchi coagulasi-positivi) sia di patogeni emergenti quale il genere Yersinia. Relativamente al genere Yersinia, sono stati indagati per tale prodotto, dati relativi a prevalenza, patogenicità e livello di contaminazione. L'attenzione verso il genere Yersinia nasce dal fatto che, negli ultimi due decenni, si è registrato un incremento nell'incidenza delle yersiniosi in diversi paesi europei, collocando la yersiniosi al terzo posto tra le zoonosi più frequentemente notificate (European Food Safety Authority, 2009).

\section{Materiali e Metodi}

Nell'ambito dei piani di sorveglianza previsti dalle ASL di competenza dei territori dell'Area Vasta della Toscana nord occidentale che comprende, 5 ASL (ASL 1 di Massa e Carrara, ASL 2 di Lucca, ASL 5 di Pisa, ASL 6 di Livorno e ASL 12 di Viareggio) sono stati analizzati 127 campioni di latte crudo bovino, nel periodo che va da maggio 2010 ad aprile 2013 , dai distributori a cui afferisce il latte prodotto dalle aziende dello stesso territorio. Nei tre anni, sono state controllate complessivamente 19 aziende produttrici di latte crudo destinato ai distributori automatici delle stesse zone.

I campioni di latte crudo, prelevati in 5 unità campionarie, per un totale di 635 campioni totali, sono stati analizzati per le ricerche previste dalla normativa vigente (Ministero della
Correspondence: Laura Gasperetti, Istituto Zooprofilattico Sperimentale delle Regioni Lazio e Toscana, Sezione di Pisa, SS Abetone e Brennero 4, 56123 Pisa (PI), Italy.

Tel. +39.050.553563 - Fax: +39.050.550615.

E-mail: laura.gasperetti@izslt.it

Key words: Yersinia, Raw milk, Foodborne pathogens.

Conflict of interests: the authors declare no potential conflict of interests.

Received for publication: 14 May 2013.

Revision received: 2 September 2013.

Accepted for publication: 30 September 2013.

This work is licensed under a Creative Commons Attribution 3.0 License (by-nc 3.0).

CC Copyright L. Gasperetti et al., 2014

Licensee PAGEPress, Italy

Italian Journal of Food Safety 2014; 3:1715

doi:10.4081/ijfs.2014.1715

Salute, 2008; Repubblica Italiana, 2007): numerazione degli Stafilococchi coagulasi positivi (ISO, 1999) e ricerca di: Campylobacter spp. (ISO, 2006), Salmonella spp. (IS0, 2002), L. monocytogenes (IS0, 2005) ed $E$. coli 0:157 (ISO, 2001) tutte eseguite con metodi ufficiali accreditati. Inoltre la presenza e la conta del genere Yersinia sono state effettuate in singolo con metodo ufficiale (ISO, 2003) e con metodo most probable number (MPN) (USDA, 2008).

L'identificazione degli isolati del genere Yersinia, sono stati identificati con test biochimici (kit miniaturizzato API 20E Biomerieux e macrometodo) e confermati con test sierologici (sieroagglutinazione rapida con sieri monovalenti per i gruppi 0:1,0:2,0:3,0:5,0:8,0:9) dal momento che la maggior parte delle infezioni umane, è sostenuta dai sierotipi 0:8, 0:9, 0:3 (biotipi 3 e 4), 0:5,27 (Asplund et al., 1998) e con metodi molecolari (PCR con protocollo interno dell'ISS) ricercando $\mathrm{i}$ geni di patogenicità, ail e yst.

\section{Risultati e Discussione}

Per quanto riguarda la ricerca dei patogeni previsti dalla normativa vigente, una sola unità campionaria su 5 di un campione è risultata positiva per E. coli 0157 , peraltro risultato non produttore di verocitotossina e solo cinque campioni sono risultati superiori ai limiti per Stafilococchi coagulasi positivi. Per la ricerca di Yersinia, un totale di 38 campioni su 127 sono risultati positivi (29,9\%): un totale di 39 isolati sono stati ottenuti; dopo conferma biochimica, un numero pari a 30 isolati sono stati 
Tabella 1. Risultati sulla identificazione dei campioni positivi per Yersinia e relative prevalenze.

\begin{tabular}{|c|c|c|c|c|}
\hline Campioni totali & Campioni positivi & $\begin{array}{c}\text { Prevalenza di Yersinia (\%) } \\
\text { e numero di isolati (n) }\end{array}$ & $\begin{array}{c}\text { Specie ritrovate e } \\
\text { numero relativo di isolati (n) }\end{array}$ & $\begin{array}{c}\text { Prevalenza } \\
\text { per specie (\%) }\end{array}$ \\
\hline \multirow{3}{*}{127} & \multirow{3}{*}{38} & \multirow{3}{*}{$29,9 \%(39)$} & Y. enterocolitica $(30)$ & 23,6 \\
\hline & & & Y. kristensenii (3) & 2,4 \\
\hline & & & Y. frederiksenii (6) & 4,7 \\
\hline
\end{tabular}

Tabella 2. Risultati sulla sierotipizzazione degli isolati di Yersinia enterocolitica e relative prevalenze.

\begin{tabular}{lccc}
\hline Isolati totali tipivati & Sierogruppo ritrovato & Frequenza (\%) \\
& Non O:1,0:2,0:3,0:5,0:8,0:9 & 21 & 70 \\
30 & $0: 5$ & 5 & 16,6 \\
& $0: 8$ & 4 & 13,3 \\
\hline
\end{tabular}

confermati come appartenenti alla specie $Y$. enterocolitica, sei alla specie $Y$. frederiksenii e tre a Y. Kristensenii (Tabella 1).

I 30 isolati di $Y$. enterocolitica sono stati sottoposti a tipizzazione sierologica e i sierogruppi prevalenti sono risultati 0:5 (16,6\%) e 0:8 (13,3\%); la maggior parte di essi (70\%) sono risultati non appartenenti ai gruppi 0:1,0:2,0:3,0:5,0:8,0:9 (Tabella 2).

Nessun isolato è risultato però patogeno, dal momento che all'analisi molecolare nessuno aveva i markers genetici ail e yst, indicativi di enteropatogenicità.

La conta di $Y$. enterocolitica, $Y$. frederiksenii e $Y$. kristensenii ha evidenziato valori compresi tra 0.03 e $24 \mathrm{MPN} / \mathrm{mL}$.

\section{Conclusioni}

Dai dati relativi al conteggio di Yersinia enterocolitica nei campioni risultati positivi, si può considerare che quando è presente, lo è a concentrazioni relativamente basse, infatti abbiamo riscontrato valori che variano tra 0.03 e $24 \mathrm{MPN} / \mathrm{mL}$, mentre in letteratura si riporta una dose infettante molto più alta, di almeno1 $0^{8}$ batteri (Fleming e Hunt, 2006).

Concludendo quindi, considerata la ristrettezza dei criteri microbiologici stabiliti dalle norme per tale prodotto, sia in considerazione dei risultati ottenuti, sia per il fatto non trascurabile che tale prodotto è da consumarsi previa bollitura, è possibile tracciare un quadro generale rassicurante sulla qualità igienico-sanitaria del latte crudo bovino prodotto e distribuito almeno in questa parte del territorio toscano.

\section{Bibliografia}

Asplund K, Hakkinen M, Okkonen T, Vanhala P, Nurmi E, 1998. Effects of growthpromoting antimicrobials on inhibition of $Y$. enterocolitica $0: 3$ by porcine ileal microflora. J Appl Microbiol 85:164-70.

European Food Safety Authority, 2009. Community Summary report on trends and sources of zoonoses and zoonotic agents in the European Union in 2007. The EFSA Journal 223:5-312.

Fleming D, Hunt D, 2006. Biological safety principles and practices. ASM Press, Washington, DC, USA.

ISO, 1999. Microbiology of food and animal feeding stuff - Horizzontal method for the numeration of coagulase-positive staphilococci (Staphylococcus aureus and other species. Part 2: Tecnique using rabbit plasma fibrinogen agar medium. Norma ISO 6888-2:1999. Organizzazione Internazionale per la Standardizzazione, Ginevra, Svizzera.

ISO, 2001. Microbiology of food and animal feeding stuffs - Horizontal method for detection of Escherichia coli 0157 . Norma ISO 16654:2001. Organizzazione Internazionale per la Standardizzazione, Ginevra, Svizzera.

ISO, 2002. Microbiology of food and animal feeding stuff. Horizontal method for the detection of Salmonella spp. Norma ISO 6579:2002/Cor 1:2004. Organizzazione Internazionale per la Standardizzazione, Ginevra, Svizzera.

ISO, 2003. Microbiology of food and animal feeding stuffs. Horizontal method for the detection of presumptive pathogenic Yersinia enterocolitica. Norma ISO 10273:2003. Organizzazione Internazionale per la Standardizzazione, Ginevra, Svizzera.

ISO, 2005. Metodo orizzontale per la ricerca e la conta di Listeria monocytogenes. Parte 1: Metodo per la ricerca. Norma ISO 112901:2005. Organizzazione Internazionale per la Standardizzazione, Ginevra, Svizzera.

ISO, 2006. Microbiology of food and animal feeding stuffs. Horizontal method for detection and enumeration of Campylobacter spp. Parte 1: detection method. Norma ISO 10272-1:2006. Organizzazione Internazionale per la Standardizzazione, Ginevra, Svizzera.

Ministero della Salute, 2008. Controlli sui distributori automatici di latte crudo per alimentazione umana. Nota ministeriale 15990-P-30/05/2008. Disponibile al sito: http://cerere.vet.unipi.it/system/files/DGSA $\mathrm{N}+15990+30+\mathrm{mag}+08+$ Distrib+latte+cru do.pdf

Repubblica Italiana, 2007. Intesa Stato-Regioni e Province Autonome di Trento e Bolzano in materia di "vendita diretta di latte crudo per l'alimentazione umana" 5/CSR 4 del 25 gennaio 2007. Disponibile al sito: http://www.governo.it/backoffice/allegati/30606-3457.pdf

USDA, 2008. USDA/FSIS-MLG Appendix 2.03 Laboratory Guidebook. Most Probable Number and Tables, 01/28/08. QD-F-Micro0004.03. Disponibile al sito: www. fsis.usda.gov/wps/wcm/connect/8872ec11d 6 a 3 - 4 f c f - 86 d f - 4 d 87 e 57780 f $5 /$ MLG_Appendix_2_03.pdf?MOD=AJPERES 